\title{
On modified $\alpha$ - $\phi$-fuzzy contractive mappings and an application to integral equations
}

\author{
Urmila Mishra', Calogero Vetro ${ }^{2}$ and Poom Kumam ${ }^{3,4^{*}}$
}

\section{"Correspondence:}

poom.kum@kmutt.ac.th

${ }^{3}$ Theoretical and Computational Science Center (TaCS), Science

Laboratory Building, Department of Mathematics, Faculty of Science, King Mongkut's University of Technology Thonburi (KMUTT), 126

Pracha Uthit Road, Bang Mod, Thung Khru, Bangkok, 10140, Thailand

${ }^{4}$ China Medical University, No. 91 , Hsueh-Shih Road, Taichung, 40402, Taiwan

Full list of author information is available at the end of the article

\begin{abstract}
We introduce the notion of a modified $\alpha$ - $\phi$-fuzzy contractive mapping and prove some results in fuzzy metric spaces for such kind of mappings. The theorems presented provide a generalization of some interesting results in the literature. Two examples and an application to integral equations are given to illustrate the usability of our theory.
\end{abstract}

MSC: $47 \mathrm{H} 10 ; 45 \mathrm{DO5}$

Keywords: $\alpha$-admissible mapping with respect to $\eta$; fixed point; modified $\alpha$ - $\phi$-fuzzy contractive mapping; integral equations

\section{Introduction}

A mathematical framework to work with 'uncertainty' or 'vagueness' was suggested by Zadeh in 1965; see [1]. In fact, the notion of a fuzzy set born as natural extension of the concept of set. Since then, this notion was used in mathematics and its applications (logic, topology, algebra, analysis, artificial intelligence, image processing and others). In particular many authors have expansively developed the theory of fuzzy metric spaces in different directions. Briefly, we say that this concept is strongly related to the concept of probabilistic metric space $[2,3]$. Also, probabilistic metric spaces are generalizations of metric spaces in which the distances take values in the class of distribution functions. Fuzzy metric spaces were introduced by Kramosil and Michalek [4]; then George and Veeramani [5] modified the concept of fuzzy metric spaces in [4] and showed that every fuzzy metric induces Hausdorff topology.

On the other hand, fixed point theorems in fuzzy metric spaces have attracted much attention. Recently Gregori and Sapena [6] introduced a kind of contractive mapping for proving Banach's contraction principle by using strong condition for completeness (G-completeness) in fuzzy metric spaces. Following this direction, in 2007, Mihet [7] proved fuzzy fixed point theorems for a Banach's contraction in $M$-complete fuzzy metric spaces. As well as she introduced some new type of contractive conditions in the setting of fuzzy metric spaces (see [8, 9]). Very recently, motivated by Samet et al. [10], the concept of $\alpha$ - $\phi$-fuzzy contractive mapping was discussed by Gopal and Vetro [11].

In view of above considerations, the fuzzy sets theory emerged as a potential area of interdisciplinary research and this is the motivation of our study. Then we introduce a

(c) 2016 Mishra et al. This article is distributed under the terms of the Creative Commons Attribution 4.0 International License (http://creativecommons.org/licenses/by/4.0/), which permits unrestricted use, distribution, and reproduction in any medium, provided you give appropriate credit to the original author(s) and the source, provide a link to the Creative Commons license, and indicate if changes were made. 
new concept of modified $\alpha$ - $\phi$-fuzzy contractive mapping and investigate the existence and uniqueness of fixed point of such kind of mappings. Finally, two examples and an application to integral equations are given to illustrate the usability of our theory.

\section{Preliminaries}

The following definitions and results will be needed in the sequel.

Definition $2.1([12])$ A binary operation $*:[0,1] \times[0,1] \rightarrow[0,1]$ is called a continuous triangular norm $(t$-norm $)$ if the following conditions hold:

(i) $*$ is associative and commutative;

(ii) $*$ is continuous;

(iii) $a * 1=a$ for all $a \in[0,1]$;

(iv) $a * b \leq c * d$, whenever $a \leq c$ and $b \leq d$, for all $a, b, c, d \in[0,1]$.

Four basic examples of continuous $t$-norms are: $a *_{1} b=\min \{a, b\}, a *_{2} b=\frac{a b}{\max \{a, b, \lambda\}}$ for $\lambda \in(0,1), a *_{3} b=a b, a *_{4} b=\max \{a+b-1,0\}$.

Definition $2.2([4])$ A fuzzy metric space is a triple $(X, M, *)$, where $X$ is a non-empty set, $*$ is a continuous $t$-norm and $M$ is a fuzzy set on $X \times X \times[0,+\infty)$ satisfying, for all $x, y \in X$ and $t>0$, the following properties:

$\left(\mathrm{K}_{1}\right) \quad M(x, y, 0)=0$

$\left(\mathrm{K}_{2}\right) M(x, y, t)=1$ iff $x=y$;

$\left(\mathrm{K}_{3}\right) M(x, y, t)=M(y, x, t)$;

$\left(\mathrm{K}_{4}\right) M(x, y, \cdot):[0,+\infty) \rightarrow[0,1]$ is left continuous;

$\left(\mathrm{K}_{5}\right) M(x, z, t+s) \geq M(x, y, t) * M(y, z, s)$, for all $z \in X$ and for all $s>0$.

George and Veeramani [5] modified the above definition and introduced a Hausdorff topology on the fuzzy metric space as follows.

Definition 2.3 ([5]) A fuzzy metric space is a triple $(X, M, *)$, where $X$ is a non-empty set, $*$ is a continuous $t$-norm and $M$ is a fuzzy set on $X \times X \times(0,+\infty)$, satisfying, for all $x, y \in X$, the following properties:

$\left(\mathrm{G}_{1}\right) M(x, y, t)>0$, for all $t>0$;

$\left(\mathrm{G}_{2}\right) M(x, y, t)=1$ for all $t>0$ iff $x=y$;

$\left(\mathrm{G}_{3}\right) M(x, y, t)=M(y, x, t)$ for all $t>0$;

$\left(\mathrm{G}_{4}\right) M(x, y, \cdot):(0,+\infty) \rightarrow[0,1]$ is continuous;

$\left(\mathrm{G}_{5}\right) M(x, z, t+s) \geq M(x, y, t) * M(y, z, s)$, for all $z \in X$ and for all $t, s>0$.

For more properties and examples of fuzzy metric spaces, the reader can refer to [4, $13-21]$.

Definition 2.4 $([5,16,22])$ Let $(X, M, *)$ be a fuzzy metric space. Then:

(i) A sequence $\left\{x_{n}\right\}$ converges to $x \in X$, that is, $\lim _{n \rightarrow+\infty} x_{n}=x$, if $\lim _{n \rightarrow+\infty} M\left(x_{n}, x, t\right)=1$ for all $t>0$.

(ii) A sequence $\left\{x_{n}\right\}$ is said to be M-Cauchy, if for each $\epsilon \in(0,1)$ and $t>0$ there exists $n_{0} \in \mathbb{N}$ such that $M\left(x_{n}, x_{m}, t\right)>1-\epsilon$ for all $m, n \geq n_{0}$. 
(iii) A sequence $\left\{x_{n}\right\}$ is said to be G-Cauchy, if $\lim _{n \rightarrow+\infty} M\left(x_{n}, x_{n+m}, t\right)=1$ for each $m \in \mathbb{N}$ and $t>0$.

Now, a fuzzy metric space $(X, M, *)$ is said to be $M$-complete (G-complete) if every $M$-Cauchy (G-Cauchy) sequence is convergent in $X$.

Definition $2.5([15,23])$ Let $(X, M, *)$ be a fuzzy metric space in the sense of George and Veeramani. The fuzzy metric $M$ is said to be triangular if the following condition holds:

$$
\left(\frac{1}{M(x, y, t)}-1\right) \leq\left(\frac{1}{M(x, z, t)}-1\right)+\left(\frac{1}{M(y, z, t)}-1\right)
$$

for all $x, y, z \in X$ and for all $t>0$.

\section{Some fixed point theorems}

In this section, we introduce the new notion of a modified $\alpha$ - $\phi$-fuzzy contractive mapping and $\alpha$-admissible mapping with respect to $\eta$ in fuzzy metric spaces.

Denote by $\Phi$ the family of all right continuous functions $\phi:[0,+\infty) \rightarrow[0,+\infty)$, with $\phi(c)<c$ for all $c>0$.

Remark 3.1 Note that for every function $\phi \in \Phi, \lim _{n \rightarrow+\infty} \phi^{n}(c)=0$, where $\phi^{n}(c)$ denotes the $n$th iterate of $\phi$.

According to [24] (see also [25]) we use the concept of $\alpha$-admissible mapping in the following form.

Definition 3.1 Let $(X, M, *)$ be a fuzzy metric space in the sense of George and Veeramani and let $\alpha, \eta: X \times X \times(0,+\infty) \rightarrow[0,+\infty)$ be two functions. We say that $T: X \rightarrow X$ is $\alpha$-admissible with respect to $\eta$ if

$$
x, y \in X, \quad \alpha(x, y, t) \geq \eta(x, y, t) \quad \Rightarrow \quad \alpha(T x, T y, t) \geq \eta(T x, T y, t) \quad \text { for all } t>0
$$

Note that if we take $\eta(x, y, t)=1$, then this definition reduces to the definition of $\alpha$-admissible mapping (Definition 3.4 of Gopal and Vetro [11]). Also, if we take $\alpha(x, y, t)=1$, then we say that $T$ is an $\eta$-subadmissible mapping.

In [11], Gopal and Vetro introduced the concept of an $\alpha$ - $\phi$-fuzzy contractive mapping in fuzzy metric spaces as follows.

Definition $3.2([11])$ Let $(X, M, *)$ be a fuzzy metric space in the sense of George and Veeramani. We say that $T: X \rightarrow X$ is an $\alpha$ - $\phi$-fuzzy contractive mapping if there exist two functions $\alpha: X \times X \times(0,+\infty) \rightarrow[0,+\infty)$ and $\phi \in \Phi$ such that, for all $x, y \in X$ and for all $t>0$, we have

$$
\alpha(x, y, t)\left(\frac{1}{M(T x, T y, t)}-1\right) \leq \phi\left(\frac{1}{M(x, y, t)}-1\right) .
$$

Motivated by the above definition we give the following generalization. 
Definition 3.3 Let $(X, M, *)$ be a fuzzy metric space in the sense of George and Veeramani. We say that $T: X \rightarrow X$ is a modified $\alpha$ - $\phi$-fuzzy contractive mapping if there exist three functions $\alpha, \eta: X \times X \times(0,+\infty) \rightarrow[0,+\infty)$ and $\phi \in \Phi$ such that, for all $x, y \in X$ and for all $t>0$, we have

$$
\alpha(x, y, t) \geq \eta(x, y, t) \quad \Rightarrow \quad \frac{1}{M(T x, T y, t)}-1 \leq \phi\left(\frac{1}{N(x, y, t)}-1\right),
$$

where $N(x, y, t)=\min \{M(x, y, t), M(x, T x, t), M(y, T y, t)\}$.

Remark 3.2 If $\eta(x, y, t)=1$ and $N(x, y, t)=M(x, y, t)$, then this definition reduces to Definition 3.2 in [11], thus it will imply the definition of the fuzzy contractive mapping given by Gregori and Sapena [6]. It follows that a fuzzy contractive mapping is a modified $\alpha-\phi$ fuzzy contractive mapping; in general the converse is not true.

Our first main result is the following theorem.

Theorem 3.1 Let $(X, M, *)$ be a G-complete fuzzy metric space in the sense of George and Veeramani. Let $T: X \rightarrow X$ be a modified $\alpha$ - $\phi$-fuzzy contractive mapping satisfying the following conditions:

(i) $T$ is $\alpha$-admissible with respect to $\eta$;

(ii) there exists $x_{0} \in X$ such that $\alpha\left(x_{0}, T x_{0}, t\right) \geq \eta\left(x_{0}, T x_{0}, t\right)$ for each $t>0$;

(iii) $T$ is continuous.

Then $T$ has a fixed point, that is, there exists $x^{*} \in X$ such that $T x^{*}=x^{*}$.

Proof Let $x_{0} \in X$ such that $\alpha\left(x_{0}, T x_{0}, t\right) \geq \eta\left(x_{0}, T x_{0}, t\right)$ for all $t>0$. Define a sequence $\left\{x_{n}\right\}$ in $X$ by $x_{n}=T x_{n-1}=T^{n} x_{0}$ for all $n \in \mathbb{N}$. Clearly, if $x_{n}=x_{n+1}$ for some $n \in \mathbb{N}$ then $x=x_{n}$ is a fixed point of $T$. Hence we suppose that $x_{n+1} \neq x_{n}$ for all $n \in \mathbb{N}$. Since $T$ is an $\alpha$-admissible mapping with respect to $\eta$ and $\alpha\left(x_{0}, T x_{0}, t\right) \geq \eta\left(x_{0}, T x_{0}, t\right)$, we deduce that

$$
\alpha\left(x_{1}, x_{2}, t\right)=\alpha\left(T x_{0}, T^{2} x_{0}, t\right) \geq \eta\left(T x_{0}, T^{2} x_{0}, t\right)=\eta\left(x_{1}, x_{2}, t\right) .
$$

Continuing this process, we get $\alpha\left(x_{n}, x_{n+1}, t\right) \geq \eta\left(x_{n}, x_{n+1}, t\right)$ for all $n \in \mathbb{N}$. Now by (2) with $x=x_{n-1}, y=x_{n}$, we get

$$
\frac{1}{M\left(T x_{n-1}, T x_{n}, t\right)} \leq \phi\left(\frac{1}{N\left(x_{n-1}, x_{n}, t\right)}\right)
$$

where

$$
\begin{aligned}
N\left(x_{n-1}, x_{n}, t\right) & =\min \left\{M\left(x_{n-1}, x_{n}, t\right), M\left(x_{n-1}, T x_{n-1}, t\right), M\left(x_{n}, T x_{n}, t\right)\right\} \\
& =\min \left\{M\left(x_{n-1}, x_{n}, t\right), M\left(x_{n-1}, x_{n}, t\right), M\left(x_{n}, x_{n+1}, t\right)\right\} \\
& =\min \left\{M\left(x_{n-1}, x_{n}, t\right), M\left(x_{n}, x_{n+1}, t\right)\right\} .
\end{aligned}
$$

It follows

$$
\frac{1}{M\left(x_{n}, x_{n+1}, t\right)}-1 \leq \phi\left(\frac{1}{\min \left\{M\left(x_{n-1}, x_{n}, t\right), M\left(x_{n}, x_{n+1}, t\right)\right\}}-1\right) .
$$


Now, if $\min \left\{M\left(x_{n-1}, x_{n}, t\right), M\left(x_{n}, x_{n+1}, t\right)\right\}=M\left(x_{n}, x_{n+1}, t\right)$ for some $n \in \mathbb{N}$, then

$$
\frac{1}{M\left(x_{n}, x_{n+1}, t\right)}-1 \leq \phi\left(\frac{1}{M\left(x_{n}, x_{n+1}, t\right)}-1\right)<\frac{1}{M\left(x_{n}, x_{n+1}, t\right)}-1
$$

which is a contradiction. Hence for all $n \in \mathbb{N}$ we have

$$
\frac{1}{M\left(x_{n}, x_{n+1}, t\right)}-1<\frac{1}{M\left(x_{n-1}, x_{n}, t\right)}-1
$$

Consequently, $M\left(x_{n}, x_{n+1}, t\right)>M\left(x_{n-1}, x_{n}, t\right)$ for all $n \in \mathbb{N}$, thus $\left\{M\left(x_{n-1}, x_{n}, t\right)\right\}$ is an increasing sequence of positive reals in $[0,1]$.

Let $s(t)=\lim _{n \rightarrow+\infty} M\left(x_{n-1}, x_{n}, t\right)$; we show that $s(t)=1$ for all $t>0$. Indeed, we assume that there exists $t_{0}>0$ such that $s\left(t_{0}\right)<1$. From

$$
\frac{1}{M\left(x_{n}, x_{n+1}, t_{0}\right)}-1 \leq \phi\left(\frac{1}{M\left(x_{n-1}, x_{n}, t_{0}\right)}-1\right)
$$

by using the right continuity of the function $\phi$, letting $n \rightarrow+\infty$, we obtain the contradiction

$$
\frac{1}{s\left(t_{0}\right)}-1 \leq \phi\left(\frac{1}{s\left(t_{0}\right)}-1\right)<\frac{1}{s\left(t_{0}\right)}-1
$$

This implies that $\lim _{n \rightarrow+\infty} M\left(x_{n-1}, x_{n}, t\right)=1$ for all $t>0$. Then, for a fixed $p \in \mathbb{N}$, we get

$$
\begin{aligned}
M\left(x_{n}, x_{n+p}, t\right) \geq & M\left(x_{n}, x_{n+1}, t / p\right) * M\left(x_{n+1}, x_{n+2}, t / p\right) * \cdots \\
& * M\left(x_{n+p-1}, x_{n+p}, t / p\right) \rightarrow 1 * \cdots * 1=1
\end{aligned}
$$

as $n \rightarrow+\infty$ and hence the sequence $\left\{x_{n}\right\}$ is $G$-Cauchy. Since $(X, M, *)$ is $G$-complete, then $\left\{x_{n}\right\}$ converges to some $x^{*} \in X$. Also, the continuity of $T$ leads to $T x_{n} \rightarrow T x^{*}$ and hence

$$
\lim _{n \rightarrow+\infty} M\left(x_{n+1}, T x^{*}, t\right)=\lim _{n \rightarrow+\infty} M\left(T x_{n}, T x^{*}, t\right)=1
$$

for all $t>0$, that is, the sequence $x_{n}$ converges to $T x^{*}$. By uniqueness of the limit, we deduce that $x^{*}=T x^{*}$; this completes the proof.

In the second theorem, we replace the continuity hypothesis with another regularity hypothesis.

Theorem 3.2 Let $(X, M, *)$ be a G-complete fuzzy metric space in the sense of George and Veeramani with $M$ triangular. Let $T: X \rightarrow X$ be a modified $\alpha-\phi$-fuzzy contractive mapping satisfying the following conditions:

(i) $T$ is $\alpha$-admissible with respect to $\eta$;

(ii) there exists $x_{0} \in X$ such that $\alpha\left(x_{0}, T x_{0}, t\right) \geq \eta\left(x_{0}, T x_{0}, t\right)$ for each $t>0$;

(iii) for any sequence $\left\{x_{n}\right\}$ in $X$ with $\alpha\left(x_{n}, x_{n+1}, t\right) \geq \eta\left(x_{n}, x_{n+1}, t\right)$ for all $n \in \mathbb{N}, t>0$ and $x_{n} \rightarrow x$ as $n \rightarrow+\infty$, then $\alpha\left(x_{n}, x, t\right) \geq \eta\left(x_{n}, x, t\right)$, for all $n \in \mathbb{N}$ and $t>0$.

Then $T$ has a fixed point, that is, there exists $x^{*} \in X$ such that $T x^{*}=x^{*}$. 
Proof By the proof of Theorem 3.1, we see that $\left\{x_{n}\right\}$ is a G-Cauchy sequence in the $G$-complete fuzzy metric space $(X, M, *)$ such that $\alpha\left(x_{n}, x_{n+1}, t\right) \geq \eta\left(x_{n}, x_{n+1}, t\right)$ for all $n \in \mathbb{N}$. Then there exists a point $x^{*}$ in $X$ such that $x_{n} \rightarrow x^{*}$ as $n \rightarrow+\infty$. Now, hypothesis (iii) of the theorem implies that

$$
\alpha\left(x_{n}, x^{*}, t\right) \geq \eta\left(x_{n}, x^{*}, t\right)
$$

for all $n \in \mathbb{N}$ and for all $t>0$.

If $x^{*} \neq T x^{*}$, that is, $M\left(x^{*}, T x^{*}, t\right)<1$ for some $t>0$, then, from (1), (3), and (2), respectively, since $M$ is triangular we write

$$
\begin{aligned}
\frac{1}{M\left(x^{*}, T x^{*}, t\right)}-1 & \leq\left(\frac{1}{M\left(x^{*}, x_{n+1}, t\right)}-1\right)+\left(\frac{1}{M\left(T x_{n}, T x^{*}, t\right)}-1\right) \\
& \leq\left(\frac{1}{M\left(x^{*}, x_{n+1}, t\right)}-1\right)+\phi\left(\frac{1}{N\left(x_{n}, x^{*}, t\right)}-1\right)
\end{aligned}
$$

Letting $n \rightarrow+\infty$, we get

$$
\begin{aligned}
N\left(x_{n}, x^{*}, t\right) & =\min \left\{M\left(x_{n}, x^{*}, t\right), M\left(x_{n}, T x_{n}, t\right), M\left(x^{*}, T x^{*}, t\right)\right\} \\
& \rightarrow \min \left\{1,1, M\left(x^{*}, T x^{*}, t\right)\right\} \\
& =M\left(x^{*}, T x^{*}, t\right)
\end{aligned}
$$

and so, to avoid contradiction with $\phi(c)<c$ for $c>0$, we conclude that

$$
\frac{1}{M\left(T x^{*}, x^{*}, t\right)}-1=0
$$

It follows that $T x^{*}=x^{*}$; this completes the proof.

Some of the following corollaries can be deduced from the above results. In particular, by taking $\eta(x, y, t)=1$ in Theorem 3.1 and Theorem 3.2 (with $M$ triangular), we have the following corollary.

Corollary 3.1 Let $(X, M, *)$ be a G-complete fuzzy metric space in the sense of George and Veeramani (with $M$ triangular). Let $T: X \rightarrow X$ be an $\alpha$-admissible mapping. Assume that there exists $\phi \in \Phi$ such that, for $x, y \in X$ and $t>0$,

$$
\alpha(x, y, t) \geq 1 \Rightarrow \frac{1}{M(T x, T y, t)}-1 \leq \phi\left(\frac{1}{N(x, y, t)}-1\right)
$$

where $N(x, y, t)=\min \{M(x, y, t), M(x, T x, t), M(y, T y, t)\}$. Also suppose that the following assertions hold:

(i) there exists $x_{0} \in X$ such that $\alpha\left(x_{0}, T x_{0}, t\right) \geq 1$ for all $t>0$;

(ii) either $T$ is continuous or for any sequence $\left\{x_{n}\right\}$ in $X$ with $\alpha\left(x_{n}, x_{n+1}, t\right) \geq 1$ for all $n \in \mathbb{N}, t>0$, and $x_{n} \rightarrow x$ as $n \rightarrow+\infty$, then $\alpha\left(x_{n}, x, t\right) \geq 1$, for all $n \in \mathbb{N}$ and $t>0$.

Then $T$ has a fixed point. 
Also, by taking $\alpha(x, y, t)=1$ in Theorem 3.1 and Theorem 3.2 (with $M$ triangular), we have the following corollary.

Corollary 3.2 Let $(X, M, *)$ be a G-complete fuzzy metric space in the sense of George and Veeramani (with $M$ triangular). Let $T: X \rightarrow X$ be an $\eta$-subadmissible mapping. Assume that there exists $\phi \in \Phi$ such that, for $x, y \in X$ and $t>0$,

$$
\eta(x, y, t) \leq 1 \quad \Rightarrow \quad \frac{1}{M(T x, T y, t)}-1 \leq \phi\left(\frac{1}{N(x, y, t)}-1\right)
$$

where $N(x, y, t)=\min \{M(x, y, t), M(x, T x, t), M(y, T y, t)\}$. Also suppose that the following assertions hold:

(i) there exists $x_{0} \in X$ such that $\eta\left(x_{0}, T x_{0}, t\right) \leq 1$ for all $t>0$;

(ii) either $T$ is continuous or for any sequence $\left\{x_{n}\right\}$ in $X$ with $\alpha\left(x_{n}, x_{n+1}, t\right) \geq 1$ for all $n \in \mathbb{N}, t>0$ and $x_{n} \rightarrow x$ as $n \rightarrow+\infty$, then $\alpha\left(x_{n}, x, t\right) \geq 1$, for all $n \in \mathbb{N}$ and $t>0$.

Then $T$ has a fixed point.

Now, we give two simple illustrative examples.

Example 3.1 Let $X=[0,+\infty)$ endowed with the fuzzy metric $M(x, y, t)=\frac{t}{t+|x-y|}$ for all $x, y \in X$ and $t>0$, and the $t$-norm $*_{3}$. Clearly $M$ is triangular. Define $T: X \rightarrow X$ by

$$
T x= \begin{cases}\frac{x^{2}}{4} & \text { if } x \in[0,1], \\ 2 & \text { if } x \in(1,+\infty)\end{cases}
$$

and $\alpha, \eta: X \times X \times(0,+\infty) \rightarrow[0,+\infty)$ by

$$
\alpha(x, y, t)= \begin{cases}1 & \text { if } x, y \in[0,1], t>0 \\ 0 & \text { otherwise }\end{cases}
$$

and $\eta(x, y, t)=1 / 3$ for all $x, y \in X$ and $t>0$. Then the existence of a fixed point for $T$ follows by an application of Theorem 3.2 with $\phi(c)=\frac{c}{2}$ for all $c \geq 0$.

Proof By completeness of $(X, d)$, where $d(x, y)=|x-y|$ for all $x, y \in X$, one deduces easily that $\left(X, M, *_{3}\right)$ is a $G$-complete metric space with $M$ triangular. We show that $T$ is an $\alpha$-admissible mapping with respect to $\eta$. Indeed, let $x, y \in X$; if $\alpha(x, y, t) \geq \eta(x, y, t)$ for all $t>0$, then $x, y \in[0,1]$. On the other hand, for all $x, y \in[0,1]$, we have $T x, T y \in[0,1]$, which implies that $\alpha(T x, T y, t) \geq \eta(T x, T y, t)$ for all $t>0$. Trivially, for every $x_{0} \in[0,1]$ we get $\alpha\left(x_{0}, T x_{0}, t\right) \geq \eta\left(x_{0}, T x_{0}, t\right)$ for all $t>0$. Now, if $\left\{x_{n}\right\}$ is a sequence in $X$ such that $\alpha\left(x_{n}, x_{n+1}, t\right) \geq \eta\left(x_{n}, x_{n+1}, t\right)$ for all $n \in \mathbb{N}$ and $x_{n} \rightarrow x$ as $n \rightarrow+\infty$, then $\left\{x_{n}\right\} \subset[0,1]$ and hence $x \in[0,1]$. This implies that $\alpha\left(x_{n}, x, t\right) \geq \eta\left(x_{n}, x, t\right)$ for all $n \in \mathbb{N}$ and $t>0$. Next, let $\alpha(x, y, t) \geq \eta(x, y, t)$ for all $t>0$, that is, $x, y \in[0,1]$. Then the contractive condition in Theorem 3.2, that is,

$$
\begin{aligned}
\frac{1}{M(T x, T y, t)}-1 & =\frac{1}{4 t}\left|x^{2}-y^{2}\right| \leq \frac{1}{2 t}|x-y| \\
& \leq \frac{1}{2 t} \max \{|x-y|,|x-T x|,|y-T y|\}
\end{aligned}
$$




$$
\begin{aligned}
& =\frac{1}{2}\left(\frac{t+\max \{|x-y|,|x-T x|,|y-T y|\}}{t}-1\right) \\
& =\frac{1}{2}\left(\frac{1}{\frac{t}{t+\max \{|x-y|,|x-T x|,|y-T y|\}}}-1\right) \\
& =\phi\left(\frac{1}{N(x, y, t)}-1\right)
\end{aligned}
$$

is always true since $x+y \leq 2$. Thus, all the hypotheses of Theorem 3.2 are satisfied and $T$ has at least a fixed point; here 0 and 2 are two fixed points of $T$.

On the other hand, $T$ is not a fuzzy contractive mapping of Gregori and Sapena [6]. In fact, for $x=1$ and $y=2$, there does not exist $k \in(0,1)$ such that

$$
\frac{1}{M(T x, T y, t)}-1=\frac{7}{4 t} \leq \frac{k}{t}=k\left(\frac{1}{M(x, y, t)}-1\right) .
$$

By a slight modification of Example 3.1, one can obtain, for instance, an example covered by Corollary 3.2 .

Example 3.2 Let $X=\left\{\frac{1}{n}, n \in \mathbb{N}\right\} \cup\{0,2\}$ endowed with the fuzzy metric $M(x, y, t)=\frac{t}{t+|x-y|}$ for all $x, y \in X$ and $t>0$, and the $t$-norm $*_{3}$. Define $T: X \rightarrow X$ by

$$
T x= \begin{cases}\frac{x^{2}}{4} & \text { if } x \in X \backslash\{2\} \\ 2 & \text { if } x=2\end{cases}
$$

and $\eta: X \times X \times(0,+\infty) \rightarrow[0,+\infty)$ by

$$
\eta(x, y, t)= \begin{cases}1 & \text { if } x, y \in X \backslash\{2\}, t>0 \\ 3 & \text { otherwise }\end{cases}
$$

Then the existence of a fixed point of $T$ follows by an application of Corollary 3.2 with $\phi(c)=\frac{c}{2}$ for all $c \geq 0$.

Proof Clearly, $T$ is an $\eta$-subadmissible continuous mapping. Shortly, let $x, y \in X$ such that $\eta(x, y, t) \leq 1$ for all $t>0$, this implies that $x, y \in X \backslash\{2\}$ and, by the definitions of $T$ and $\eta$, we have $T x, T y \in X \backslash\{2\}$ and $\eta(T x, T y, t)=1$ for all $t>0$, that is, $T$ is $\eta$-subadmissible. Further, for every $x_{0} \in X \backslash\{2\}$ we get $\eta\left(x_{0}, T x_{0}, t\right)=1$ for all $t>0$.

Next, let $\eta(x, y, t) \leq 1$ for all $t>0$, that is, $x, y \in X \backslash\{2\}$. By using the same argument as in Example 3.1, the contractive condition in Corollary 3.2 is always true since $x+y \leq 2$. Thus, all the hypotheses of Corollary 3.2 are satisfied; again 0 and 2 are two fixed points of $T$.

Next, for establishing the uniqueness of fixed point of modified $\alpha$ - $\phi$-fuzzy contractive mapping, we will consider the following hypothesis:

(H) for all $x, y \in X$ and for all $t>0$ there exists $z \in X$ such that $\alpha(x, z, t) \geq \eta(x, z, t)$, $\alpha(y, z, t) \geq \eta(y, z, t)$ and $\lim _{n \rightarrow+\infty} M\left(T^{n-1} z, T^{n} z, t\right)=1$.

Theorem 3.3 Adding hypothesis $(\mathrm{H})$ in Theorem 3.1 (Theorem 3.2), one obtains that $x^{*}$ is the unique fixed point of $T$ provided that $\phi \in \Phi$ is non-decreasing. 
Proof Assume that $x^{*}$ and $y^{*}$ are two fixed points of $T$. If $\alpha\left(x^{*}, y^{*}, t\right) \geq \eta\left(x^{*}, y^{*}, t\right)$, then by condition (2), we get $x^{*}=y^{*}$. Suppose $\alpha\left(x^{*}, y^{*}, t\right)<\eta\left(x^{*}, y^{*}, t\right)$, then from hypothesis (H) there exists $z \in X$ such that

$$
\alpha\left(x^{*}, z, t\right) \geq \eta\left(x^{*}, z, t\right) \quad \text { and } \quad \alpha\left(y^{*}, z, t\right) \geq \eta\left(y^{*}, z, t\right) .
$$

Since $T$ is $\alpha$-admissible with respect to $\eta$, then we have

$$
\alpha\left(x^{*}, T^{n} z, t\right) \geq \eta\left(x^{*}, T^{n} z, t\right)
$$

for all $n \in \mathbb{N}$ and for all $t>0$. Now we prove that $M\left(x^{*}, T^{n} z, t\right) \rightarrow 1$, as $n \rightarrow+\infty$, for all $t>0$. Reasoning by absurd, we suppose that there exists $t>0$ such that $\lim _{n \rightarrow+\infty} M\left(x^{*}, T^{n} z, t\right)<1$. Then, from (2) and (5), we get

$$
\begin{aligned}
\frac{1}{M\left(x^{*}, T^{n} z, t\right)}-1 & =\frac{1}{M\left(T x^{*}, T\left(T^{n-1} z\right), t\right)}-1 \\
& \leq \phi\left(\frac{1}{N\left(x^{*}, T^{n-1} z, t\right)}-1\right),
\end{aligned}
$$

where

$$
\begin{aligned}
N\left(x^{*}, T^{n-1} z, t\right) & =\min \left\{M\left(x^{*}, T^{n-1} z, t\right), M\left(x^{*}, T x^{*}, t\right), M\left(T^{n-1} z, T\left(T^{n-1}\right) z, t\right)\right\} \\
& =\min \left\{M\left(x^{*}, T^{n-1} z, t\right), M\left(x^{*}, T x^{*}, t\right), M\left(T^{n-1} z, T^{n} z, t\right)\right\} \\
& =\min \left\{M\left(x^{*}, T^{n-1} z, t\right), 1, M\left(T^{n-1} z, T^{n} z, t\right)\right\} .
\end{aligned}
$$

Let $n_{0} \in \mathbb{N}$ such that $M\left(x^{*}, T^{n} z, t\right) \leq M\left(T^{n} z, T^{n+1} z, t\right)$ for all $n \geq n_{0}$. Therefore, for all $n>n_{0}$, we obtain

$$
\begin{aligned}
\frac{1}{M\left(x^{*}, T^{n} z, t\right)}-1 & =\frac{1}{M\left(T x^{*}, T\left(T^{n-1} z\right), t\right)}-1 \\
& \leq \phi\left(\frac{1}{\min \left\{M\left(x^{*}, T^{n-1} z, t\right), 1, M\left(T^{n-1} z, T^{n} z, t\right)\right\}}-1\right) \\
& =\phi\left(\frac{1}{M\left(x^{*}, T^{n-1} z, t\right)}-1\right) .
\end{aligned}
$$

This implies that

$$
\begin{aligned}
\frac{1}{M\left(x^{*}, T^{n} z, t\right)}-1 & =\frac{1}{M\left(T x^{*}, T\left(T^{n-1} z\right), t\right)}-1 \\
& \leq \phi^{n-n_{0}}\left(\frac{1}{M\left(x^{*}, T^{n_{0}} z, t\right)}-1\right) .
\end{aligned}
$$

Then, letting $n \rightarrow+\infty$, we get $T^{n} z \rightarrow x^{*}$. Similarly one can obtain $T^{n} z \rightarrow y^{*}$, as $n \rightarrow+\infty$. Consequently, we deduce that $x^{*}=y^{*}$; the uniqueness is proved.

The following classes of functions are used in our subsequent results. Let

$$
\Psi=\{\psi:[0,+\infty) \rightarrow[0,+\infty) \text { such that } \psi \text { is non-decreasing and continuous }\}
$$


and

$\Phi_{1}=\{\phi:[0,+\infty) \rightarrow[0,+\infty)$ such that $\phi$ is lower semicontinuous $\}$

where $\psi(r)=\phi(r)=0$ if and only if $r=0$.

Theorem 3.4 Let $(X, M, *)$ be a G-complete fuzzy metric space and let $T: X \rightarrow X$ be an $\alpha$-admissible mapping with respect to $\eta$. Assume that there exist $\psi \in \Psi$ and $\phi \in \Phi_{1}$ such that, for $x, y \in X$ and $t>0$,

$\alpha(x, T x, t) \alpha(y, T y, t) \geq \eta(x, T x, t) \eta(y, T y, t)$

$$
\Rightarrow \quad \psi\left(\frac{1}{M(T x, T y, t)}-1\right) \leq \psi\left(\frac{1}{M(x, y, t)}-1\right)-\phi\left(\frac{1}{M(x, y, t)}-1\right) .
$$

Also suppose that the following conditions hold:

(i) there exists $x_{0} \in X$ such that $\alpha\left(x_{0}, T x_{0}, t\right) \geq \eta\left(x_{0}, T x_{0}, t\right)$ for all $t>0$;

(ii) either $T$ is continuous or for any sequence $\left\{x_{n}\right\}$ in $X$ with $\alpha\left(x_{n}, x_{n+1}, t\right) \geq \eta\left(x_{n}, x_{n+1}, t\right)$ for all $n \in \mathbb{N}, t>0$, and $x_{n} \rightarrow x$ as $n \rightarrow+\infty$, then $\alpha\left(x_{n}, x, t\right) \geq \eta\left(x_{n}, x, t\right)$ and $\alpha(x, T x, t) \geq \eta(x, T x, t)$, for all $n \in \mathbb{N}$ and $t>0$.

Then $T$ has a fixed point.

Proof Let $x_{0}$ in $X$ such that $\alpha\left(x_{0}, T x_{0}, t\right) \geq \eta\left(x_{0}, T x_{0}, t\right)$. Define a sequence $\left\{x_{n}\right\}$ in $X$ such that $x_{n}=T x_{n-1}=T^{n} x_{0}$ for all $n \in \mathbb{N}$. If $x_{n+1}=x_{n}$ for some $n \in X$, then $x=x_{n}$ is a fixed point for $T$ and the result is proved in this case. Now, we assume that $x_{n} \neq x_{n+1}$ for all $n \in \mathbb{N}$. Since $T$ is $\alpha$-admissible with respect to $\eta$ and $\alpha\left(x_{0}, T x_{0}, t\right) \geq \eta\left(x_{0}, T x_{0}, t\right)$, we deduce that

$$
\alpha\left(x_{1}, x_{2}, t\right)=\alpha\left(T x_{0}, T^{2} x_{0}, t\right) \geq \eta\left(T x_{0}, T^{2} x_{0}, t\right)=\eta\left(x_{1}, x_{2}, t\right) .
$$

Continuing this process we get

$$
\alpha\left(x_{n}, x_{n+1}, t\right) \geq \eta\left(x_{n}, x_{n+1}, t\right)
$$

for all $n \in \mathbb{N}$. Clearly,

$$
\alpha\left(x_{n-1}, T x_{n-1}, t\right) \alpha\left(x_{n}, T x_{n}, t\right) \geq \eta\left(x_{n-1}, T x_{n-1}, t\right) \eta\left(x_{n}, T x_{n}, t\right) .
$$

Now by (6) with $x=x_{n-1}, y=x_{n}$, we have

$$
\psi\left(\frac{1}{M\left(T x_{n-1}, T x_{n}, t\right)}-1\right) \leq \psi\left(\frac{1}{M\left(x_{n-1}, x_{n}, t\right)}-1\right)-\phi\left(\frac{1}{M\left(x_{n-1}, x_{n}, t\right)}-1\right)
$$

which implies that

$$
\psi\left(\frac{1}{M\left(x_{n}, x_{n+1}, t\right)}-1\right) \leq \psi\left(\frac{1}{M\left(x_{n-1}, x_{n}, t\right)}-1\right)-\phi\left(\frac{1}{M\left(x_{n-1}, x_{n}, t\right)}-1\right)
$$

If $M\left(x_{n-1}, x_{n}, t\right)=1$, then $M\left(x_{n}, x_{n+1}, t\right)=1$ too. Otherwise, if $M\left(x_{n-1}, x_{n}, t\right)<1$, then

$$
\psi\left(\frac{1}{M\left(x_{n}, x_{n+1}, t\right)}-1\right)<\psi\left(\frac{1}{M\left(x_{n-1}, x_{n}, t\right)}-1\right)
$$


and, since $\psi$ is non-decreasing, we get $M\left(x_{n}, x_{n+1}, t\right) \geq M\left(x_{n-1}, x_{n}, t\right)$, for all $n \in \mathbb{N}$. Thus, $\left\{M\left(x_{n-1}, x_{n}, t\right)\right\}$ is a non-decreasing sequence of positive reals in $[0,1]$. Let $s(t)=$ $\lim _{n \rightarrow+\infty} M\left(x_{n-1}, x_{n}, t\right)$. Now we show that $s(t)=1$ for all $t>0$. If not, then there exists $t>0$ such that $s(t)<1$. Therefore, from the above inequality, on taking $n \rightarrow+\infty$, we obtain

$$
\psi\left(\frac{1}{s(t)}-1\right) \leq \psi\left(\frac{1}{s(t)}-1\right)-\phi\left(\frac{1}{s(t)}-1\right)
$$

that is a contradiction and so we get $\lim _{n \rightarrow+\infty} M\left(x_{n-1}, x_{n}, t\right)=1$, for all $t>0$. Next, for a fixed $p \in \mathbb{N}$, we have

$$
\begin{aligned}
M\left(x_{n}, x_{n+p}, t\right) \geq & M\left(x_{n}, x_{n+1}, t / p\right) * M\left(x_{n+1}, x_{n+2}, t / p\right) \\
& * \cdots * M\left(x_{n+p-1}, x_{n+p}, t / p\right) \\
\rightarrow & 1 * \cdots * 1=1
\end{aligned}
$$

as $n \rightarrow+\infty$ and thus $\left\{x_{n}\right\}$ is a $G$-Cauchy sequence. Since $(X, M, *)$ is $G$-complete, therefore $\left\{x_{n}\right\}$ converges to $x^{*}$ for some $x^{*} \in X$. Now, we distinguish two cases.

Case 1. $T$ is continuous. Then this implies that $T x_{n} \rightarrow T x^{*}$ and so

$$
\lim _{n \rightarrow+\infty} M\left(x_{n+1}, T x^{*}, t\right)=\lim _{n \rightarrow+\infty} M\left(T x_{n}, T x^{*}, t\right)=1
$$

for all $t>0$, that is, $x_{n} \rightarrow T x^{*}$. By the uniqueness of the limit, we get $x^{*}=T x^{*}$, that is, $x^{*}$ is a fixed point of $T$.

Case 2. For any sequence $\left\{x_{n}\right\}$ in $X$ with $\alpha\left(x_{n}, x_{n+1}, t\right) \geq \eta\left(x_{n}, x_{n+1}, t\right)$ for all $n \in \mathbb{N}, t>0$, and $x_{n} \rightarrow x^{*}$ as $n \rightarrow+\infty$, then $\alpha\left(x_{n}, x^{*}, t\right) \geq \eta\left(x_{n}, x^{*}, t\right)$ and $\alpha\left(x^{*}, T x^{*}, t\right) \geq \eta\left(x^{*}, T x^{*}, t\right)$, for all $n \in \mathbb{N}$ and $t>0$. In this case, we get easily

$$
\alpha\left(x_{n}, x_{n+1}, t\right) \alpha\left(x^{*}, T x^{*}, t\right) \geq \eta\left(x_{n}, x_{n+1}, t\right) \eta\left(x^{*}, T x^{*}, t\right) .
$$

Now by (6), we have

$$
\begin{aligned}
\psi\left(\frac{1}{M\left(x_{n+1}, T x^{*}, t\right)}-1\right) & =\psi\left(\frac{1}{M\left(T x_{n}, T x^{*}, t\right)}-1\right) \\
& \leq \psi\left(\frac{1}{M\left(x_{n}, x^{*}, t\right)}-1\right)-\phi\left(\frac{1}{M\left(x_{n}, x^{*}, t\right)}-1\right) .
\end{aligned}
$$

If $M\left(x_{n}, x^{*}, t\right)=1$, then $M\left(x_{n+1}, T x^{*}, t\right)=1$ too. Otherwise, if $M\left(x_{n}, x^{*}, t\right)<1$, then

$$
\psi\left(\frac{1}{M\left(x_{n+1}, T x^{*}, t\right)}-1\right)<\psi\left(\frac{1}{M\left(x_{n}, x^{*}, t\right)}-1\right) .
$$

This implies that $M\left(x_{n+1}, T x^{*}, t\right) \geq M\left(x_{n}, x^{*}, t\right) \rightarrow 1$ as $n \rightarrow+\infty$ for all $t>0$ and so $x^{*}=$ $T x^{*}$.

By taking $\eta(x, y, t)=1$ in Theorem 3.4, we deduce the following corollary.

Corollary 3.3 Let $(X, M, *)$ be a G-complete fuzzy metric space and let $T: X \rightarrow X$ be an $\alpha$-admissible mapping. Assume that there exist $\psi \in \Psi$ and $\phi \in \Phi_{1}$ such that, for $x, y \in X$ 
and $t>0$,

$$
\begin{aligned}
& \alpha(x, T x, t) \alpha(y, T y, t) \geq 1 \\
& \quad \Rightarrow \quad \psi\left(\frac{1}{M(T x, T y, t)}-1\right) \leq \psi\left(\frac{1}{M(x, y, t)}-1\right)-\phi\left(\frac{1}{M(x, y, t)}-1\right) .
\end{aligned}
$$

Also suppose that the following conditions hold:

(i) there exists $x_{0} \in X$ such that $\alpha\left(x_{0}, T x_{0}, t\right) \geq 1$ for all $t>0$;

(ii) either $T$ is continuous or for any sequence $\left\{x_{n}\right\}$ in $X$ with $\alpha\left(x_{n}, x_{n+1}, t\right) \geq 1$ for all $n \in \mathbb{N}, t>0$ and $x_{n} \rightarrow x$ as $n \rightarrow+\infty$, then $\alpha\left(x_{n}, x, t\right) \geq 1$ and $\alpha(x, T x, t) \geq 1$, for all $n \in \mathbb{N}$ and $t>0$.

Then $T$ has a fixed point.

Also, by taking $\alpha(x, y, t)=1$ in Theorem 3.4 we deduce the following corollary.

Corollary 3.4 Let $(X, M, *)$ be a G-complete fuzzy metric space and let $T: X \rightarrow X$ be an $\eta$-subadmissible mapping. Assume that there exist $\psi \in \Psi$ and $\phi \in \Phi_{1}$ such that, for $x, y \in X$ and $t>0$,

$$
\begin{aligned}
& \eta(x, T x, t) \eta(y, T y, t) \leq 1 \\
& \quad \Rightarrow \quad \psi\left(\frac{1}{M(T x, T y, t)}-1\right) \leq \psi\left(\frac{1}{M(x, y, t)}-1\right)-\phi\left(\frac{1}{M(x, y, t)}-1\right) .
\end{aligned}
$$

Also suppose that the following conditions hold:

(i) there exists $x_{0} \in X$ such that $\eta\left(x_{0}, T x_{0}, t\right) \leq 1$ for all $t>0$;

(ii) either $T$ is continuous or for any sequence $\left\{x_{n}\right\}$ in $X$ with $\eta\left(x_{n}, x_{n+1}, t\right) \leq 1$ for all $n \in \mathbb{N}, t>0$, and $x_{n} \rightarrow x$ as $n \rightarrow+\infty$, then $\eta\left(x_{n}, x, t\right) \leq 1$ and $\eta(x, T x, t) \leq 1$, for all $n \in \mathbb{N}$ and $t>0$.

Then $T$ has a fixed point.

\section{Application to integral equation}

Inspired by $[3,26]$, we present an application of fixed point techniques to integral equations, by using our theory. Precisely, we discuss the existence of solution for the following general integral equation:

$$
x(r)=g(r)+\int_{0}^{r} K(r, s, x(s)) d s \text { for all } r \in[0, I]
$$

where $I>0$.

Let $C([0, I], \mathbb{R})$ be the Banach space of all continuous functions defined on $[0, I]$ endowed with the supremum norm

$$
\|x\|=\sup _{r \in[0, I]}|x(r)|, \quad x \in C([0, I], \mathbb{R}),
$$

and the induced metric

$$
d(x, y)=\sup _{r \in[0, I]}|x(r)-y(r)|, \quad x, y \in C([0, I], \mathbb{R}) .
$$


First of all, according to [5], let $a * b=a b$ for all $a, b \in[0,1]$ (in short $*_{3}$ ), and consider the fuzzy metric

$$
M_{d}(x, y, t)=\frac{t}{t+d(x, y)}, \quad t>0, x, y \in C([0, I], \mathbb{R}) .
$$

This $M_{d}$ is called the standard fuzzy metric induced by $d$. The topologies induced by the standard fuzzy metric and the corresponding metric are the same; see [5]. In view of (8), the Banach space $C([0, I], \mathbb{R})$ may be regarded as a fuzzy Banach space. Indeed the space $\left(C([0, I], \mathbb{R}), M_{d}, *_{3}\right)$ is the $G$-complete fuzzy metric space induced by the Banach space $C([0, I], \mathbb{R})$; see $[2,4]$ for more details.

Thus, Banach spaces and fuzzy Banach spaces are strongly related to each other; see for instance [27]. Without going in details, there are examples of fuzzy Banach spaces which are not Banach spaces. Therefore, fuzzy Banach spaces cover a broad spectrum of categories than the corresponding Banach spaces. This is a sufficient motivation to develop an application in a fuzzy setting.

Now, we discuss the existence of a solution for the integral equation (7).

Theorem 4.1 Let $T: C([0, I], \mathbb{R}) \rightarrow C([0, I], \mathbb{R})$ be the integral operator given by

$$
T x(r)=g(r)+\int_{0}^{r} K(r, s, x(s)) d s, \quad g \in C([0, I], \mathbb{R}),
$$

where $K \in C([0, I] \times[0, I] \times \mathbb{R}, \mathbb{R})$ satisfies the following condition:

(i) there exists $f:[0, I] \times[0, I] \rightarrow[0,+\infty)$ such that, for all $r \in[0, I], f(r, \cdot) \in L^{1}([0, I], \mathbb{R})$, and, for all $x, y \in C([0, I], \mathbb{R})$ and for all $r, s \in[0, I]$, we get

$$
\begin{aligned}
& |K(r, s, x(s))-K(r, s, y(s))| \\
& \quad \leq f(r, s) \max \{|x(s)-y(s)|,|x(s)-T x(s)|,|y(s)-T y(s)|\},
\end{aligned}
$$

where $\int_{0}^{r} f(r, s) d s$ is bounded on $[0, I]$ and $\sup _{r \in[0, I]} \int_{0}^{r} f(r, s) d s \leq \lambda<1$.

Then the integral equation (7) has a solution $x^{*} \in C([0, I], \mathbb{R})$.

Proof For all $x, y \in C([0, I], \mathbb{R})$, we get

$$
\begin{aligned}
& |T x(r)-T y(r)| \\
& \quad \leq \int_{0}^{r}|K(r, s, x(s))-K(r, s, y(s))| d s \\
& \quad \leq \int_{0}^{r} f(r, s) \max \{|x(s)-y(s)|,|x(s)-T x(s)|,|y(s)-T y(s)|\} d s \\
& \quad \leq \max \{d(x, y), d(x, T x), d(y, T y)\} \int_{0}^{r} f(r, s) d s \\
& \quad \leq \lambda \max \{d(x, y), d(x, T x), d(y, T y)\} .
\end{aligned}
$$

It follows that

$$
d(T x, T y) \leq \lambda \max \{d(x, y), d(x, T x), d(y, T y)\} .
$$


In view of (8), for all $x, y \in C([0, I], \mathbb{R})$ and $t>0$, we can write

$$
\begin{aligned}
N_{d}(x, y, t) & =\min \left\{\frac{t}{t+d(x, y)}, \frac{t}{t+d(x, T x)}, \frac{t}{t+d(y, T y)}\right\} \\
& =\frac{t}{t+\max \{d(x, y), d(x, T x), d(y, T y)\}}
\end{aligned}
$$

and so

$$
\begin{aligned}
\frac{1}{N_{d}(x, y, t)}-1 & =\frac{t+\max \{d(x, y), d(x, T x), d(y, T y)\}}{t}-1 \\
& =\frac{\max \{d(x, y), d(x, T x), d(y, T y)\}}{t} .
\end{aligned}
$$

Consequently, after routine calculations, we obtain

$$
\begin{aligned}
\frac{1}{M_{d}(T x, T y, t)}-1 & =\frac{d(T x, T y)}{t} \\
& \leq \lambda \frac{\max \{d(x, y), d(x, T x), d(y, T y)\}}{t} \\
& =\lambda\left(\frac{1}{N_{d}(x, y, t)}-1\right) .
\end{aligned}
$$

This implies that (4) holds true with $\phi(c)=\lambda c$ for all $c \geq 0$ and $\alpha(x, y, t)=1$ for all $x, y \in$ $C([0, I], \mathbb{R})$ and $t>0$.

As mentioned above, since $C([0, I], \mathbb{R})$ is complete, then the fuzzy metric space $(C([0, I]$, $\left.\mathbb{R}), M_{d}, *_{3}\right)$ is $G$-complete with $M$ triangular. The other hypotheses of Corollary 3.1 are immediately satisfied and hence we deduce that the operator $T$ has a fixed point $x^{*} \in$ $C([0, I], \mathbb{R})$, which is a solution of the integral equation (7).

Competing interests

The authors declare that they have no competing interests.

\section{Authors' contributions}

All authors contributed equally and significantly in writing this article. All authors read and approved the final manuscript.

\section{Author details}

${ }^{1}$ Department of Mathematics, Vishwavidyalaya Engineering College, Lakhanpur-Ambikapur, Chhattisgarh, India. ${ }^{2}$ Department of Mathematics and Computer Sciences, University of Palermo, Via Archirafi 34, Palermo, 90123, Italy. ${ }^{3}$ Theoretical and Computational Science Center (TaCS), Science Laboratory Building, Department of Mathematics, Faculty of Science, King Mongkut's University of Technology Thonburi (KMUTT), 126 Pracha Uthit Road, Bang Mod, Thung Khru, Bangkok, 10140, Thailand. ${ }^{4}$ China Medical University, No. 91, Hsueh-Shih Road, Taichung, 40402, Taiwan.

\section{Acknowledgements}

This research project was supported by the Theoretical and Computational Science (TaCS) Center.

Received: 11 September 2015 Accepted: 3 February 2016 Published online: 18 February 2016

\section{References}

1. Zadeh, LA: Fuzzy sets. Inf. Control 8, 338-353 (1965)

2. Hadzić, O, Ovcin, Z: Fixed point theorems in fuzzy metric and probabilistic metric spaces. Zb. Rad. Prir.-Mat. Fak., Ser. Mat. 24(2), 197-209 (1994)

3. Shisheng, Z: On the theory of probabilistic metric spaces with applications. Acta Math. Sin. Engl. Ser. 1, 366-377 (1985)

4. Kramosil, I, Michalek, J: Fuzzy metric and statistical metric spaces. Kybernetika 11, 336-344 (1975)

5. George, A, Veeramani, P: On some results in fuzzy metric spaces. Fuzzy Sets Syst. 64, 395-399 (1994)

6. Gregori, V, Sapena, A: On fixed point theorems in fuzzy metric spaces. Fuzzy Sets Syst. 125, 245-253 (2002) 
7. Mihet, D: A Banach contraction theorem in fuzzy metric spaces. Fuzzy Sets Syst. 144, 431-439 (2004)

8. Mihet, D: On fuzzy contractive mappings in fuzzy metric spaces. Fuzzy Sets Syst. 158, 915-921 (2007)

9. Mihet, D: Fuzzy $\psi$-contractive mappings in non-Archimedean fuzzy metric spaces. Fuzzy Sets Syst. 159, 739-744 (2008)

10. Samet, B, Vetro, C, Vetro, P: Fixed point theorems for $\phi$-contractive type mappings. Nonlinear Anal. 75, 2154-2165 (2012)

11. Gopal, D, Vetro, C: Some new fixed point theorems in fuzzy metric spaces. Iran. J. Fuzzy Syst. 11(3), $95-107$ (2014)

12. Schweizer, B, Sklar, A: Statistical metric spaces. Pac. J. Math. 10, 385-389 (1960)

13. Altun, I: Some fixed point theorems for single and multi valued mappings on ordered non-Archimedean fuzzy metric spaces. Iran. J. Fuzzy Syst. 7, 91-96 (2010)

14. Cho, YJ: Fixed points in fuzzy metric spaces. J. Fuzzy Math. 5, 949-962 (1997)

15. Di Bari, C, Vetro, C: A fixed point theorem for a family of mappings in a fuzzy metric space. Rend. Circ. Mat. Palermo 52, 315-321 (2003)

16. Grabiec, M: Fixed points in fuzzy metric spaces. Fuzzy Sets Syst. 27, 385-389 (1988)

17. Gregori, V, Morillas, S, Sapena, A: Examples of fuzzy metrics and applications. Fuzzy Sets Syst. 170, $95-111$ (2011)

18. Kaleva, O, Seikkala, S: On fuzzy metric spaces. Fuzzy Sets Syst. 12, 215-229 (1984)

19. Mishra, U, Ranadive, AS, Gopal, D: Fixed point theorems via absorbing maps. Thai J. Math. 6, 49-60 (2008)

20. Murthy, PP, Mishra, U, Rashmi, Vetro, C: Generalized $(\phi, \psi)$-weak contractions involving $(f ; g)$-reciprocally continuous maps in fuzzy metric spaces. Ann. Fuzzy Math. Inform. 5, 45-57 (2013)

21. Vetro, C, Vetro, P: Common fixed points for discontinuous mappings in fuzzy metric spaces. Rend. Circ. Mat. Palermo 57, 295-303 (2008)

22. Vasuki, R, Veeramani, P: Fixed point theorems and Cauchy sequences in fuzzy metric spaces. Fuzzy Sets Syst. 135 415-417 (2003)

23. Di Bari, C, Vetro, C: Fixed points, attractors and weak fuzzy contractive mappings in a fuzzy metric space. J. Fuzzy Math. 13(4), 973-982 (2005)

24. Salimi, P, Latif, A, Hussain, N: Modified $\alpha$ - $\psi$-contractive mappings with applications. Fixed Point Theory Appl. 2013 Article ID 151 (2013)

25. Hussain, N, Salimi, P, Latif, A: Fixed point results for single and set-valued $\alpha-\eta$ - $\psi$-contractive mappings. Fixed Point Theory Appl. 2013, Article ID 212 (2013)

26. Gopal, D, Abbas, M, Vetro, C: Some new fixed point theorems in Menger PM-spaces with application to Volterra type integral equation. Appl. Math. Comput. 232, 955-967 (2014)

27. Saadati, R, Vaezpour, SM: Some results on fuzzy Banach spaces. J. Appl. Math. Comput. 17, 475-484 (2005)

\section{Submit your manuscript to a SpringerOpen ${ }^{\ominus}$ journal and benefit from:}

- Convenient online submission

- Rigorous peer review

- Immediate publication on acceptance

- Open access: articles freely available online

- High visibility within the field

- Retaining the copyright to your article 